\title{
Network Pharmacology Reveals That Resveratrol Can Alleviate COVID-19-Related Hyperinflammation
}

\author{
Zijian Xiao, Qing Ye, Xiaomei Duan, and Tao Xiang \\ The First Affiliated Hospital, Department of Neurology, Hengyang Medical School, University of South China, Hengyang, \\ Hunan, China \\ Correspondence should be addressed to Tao Xiang; xiangtao2019@sina.com
}

Received 14 June 2021; Revised 17 August 2021; Accepted 26 August 2021; Published 23 September 2021

Academic Editor: Luigi Mario Castello

Copyright (c) 2021 Zijian Xiao et al. This is an open access article distributed under the Creative Commons Attribution License, which permits unrestricted use, distribution, and reproduction in any medium, provided the original work is properly cited.

\begin{abstract}
Hyperinflammation is related to the development of COVID-19. Resveratrol is considered an anti-inflammatory and antiviral agent. Herein, we used a network pharmacological approach and bioinformatic gene analysis to explore the pharmacological mechanism of Resveratrol in COVID-19 therapy. Potential targets of Resveratrol were obtained from public databases. SARSCoV-2 differentially expressed genes (DEGs) were screened out via bioinformatic analysis Gene Expression Omnibus (GEO) datasets GSE147507, followed by Gene Ontology (GO) and Kyoto Encyclopedia of Genes and Genomes (KEGG) pathway enrichment analysis; then, protein-protein interaction network was constructed. The common targets, GO terms, and KEGG pathways of Resveratrol targets and SARS-CoV-2 DEGs were confirmed. KEGG Mapper queried the location of common targets in the key pathways. A notable overlap of the GO terms and KEGG pathways between Resveratrol targets and SARSCoV-2 DEGs was revealed. The shared targets between Resveratrol targets and SARS-CoV-2 mainly involved the IL-17 signaling pathway, NF-kappa B signaling pathway, and TNF signaling pathway. Our study uncovered that Resveratrol is a promising therapeutic candidate for COVID-19 and we also revealed the probable key targets and pathways involved. Ultimately, we bring forward new insights and encourage more studies on Resveratol to benefit COVID-19 patients.
\end{abstract}

\section{Introduction}

The outbreak of coronavirus disease 2019 (COVID-19) has caused a global health emergency. People worldwide are still being challenged by the enhanced infection risk as coronavirus 2 (SARS-CoV-2) is rapidly spreading [1]. Hence, researchers urgently need to accelerate clinical trials of any possible effective and tolerable drug that may reduce the mortality rate in severe SARS-CoV-2 pneumonia patients.

Severe COVID-19 patients often demonstrate acute respiratory distress syndrome (ARDS). Proinflammatory cytokines in the blood were found to be upregulated in COVID-19 patients, including interleukin- (IL-) 1, IL-6, tumor necrosis factor $(T N F)$, and interferon $\gamma$. Studies suggest that a subgroup of patients with severe COVID-19 might have acquired cytokine storm syndrome [1]. Accumulated evidence has confirmed that excessive inflammation, oxidation, and an exaggerated immune response may play an important role during a cytokine storm and subsequent progression to acute lung injury (ALI)/ARDS and often death $[1,2]$. Approved therapies to alleviate hyperinflammation and improve the prognosis of severe COVID-19 patients are recommended.

Resveratrol (PubChem CID: 445154) is a phytoalexin that can be extracted from grapes and a wide range of plants and possesses antioxidant and potential chemopreventive properties. Resveratrol displays anti-inflammatory effects via regulating immune cells and interfering with the synthesis of proinflammatory cytokines [3-5]. Resveratrol also acts as an antiviral agent through different mechanisms of action. Resveratrol has been shown to inhibit various viruses, including respiratory syncytial virus, influenza virus, human metapneumonia virus, Epstein-Barr virus, enterovirus, and HIV [6-8].

Our previous study predicted that astragaloside IV could alleviate hyperinflammation in COVID-19 using network 


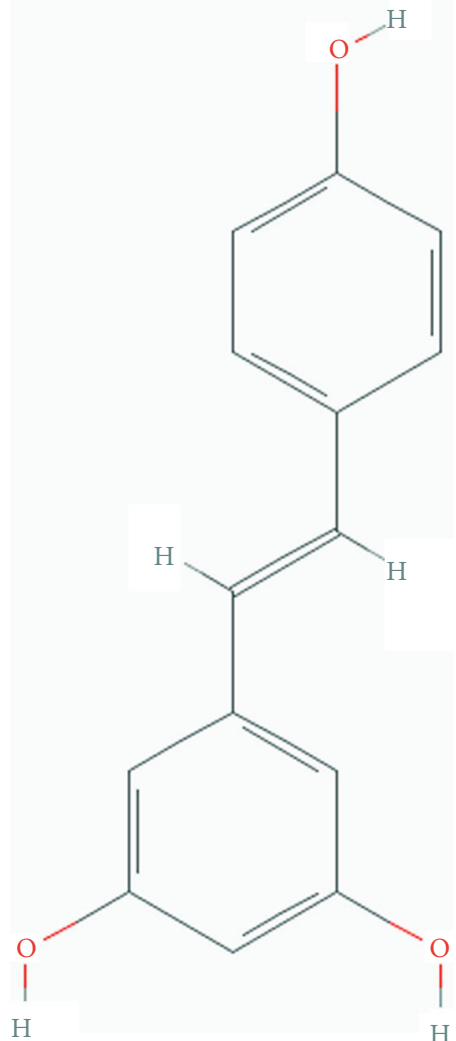

(a)

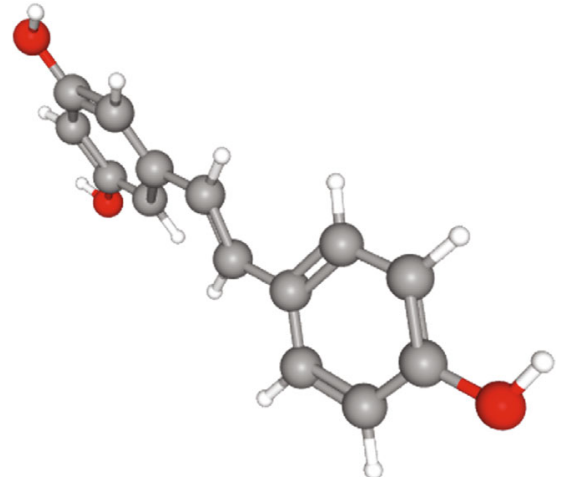

(b)

FIGURE 1: Molecular structure of Resveratrol: (a) 2D molecular structure; (b) 3D molecular structure.

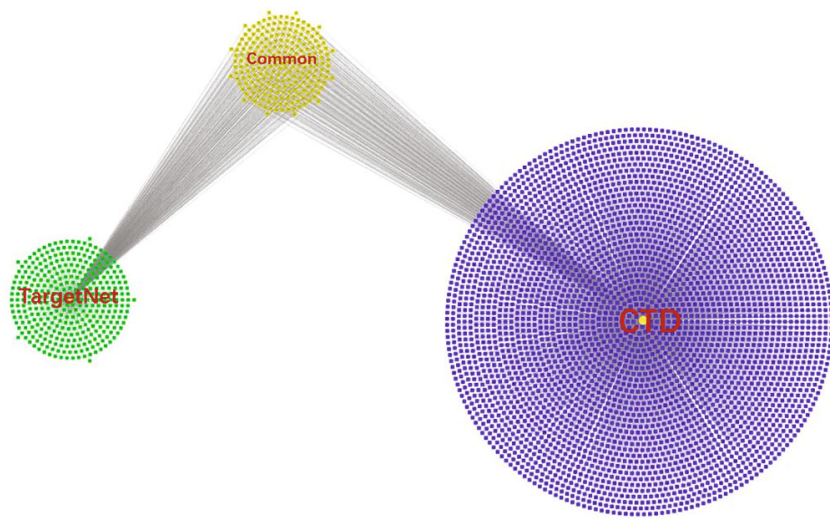

(a)

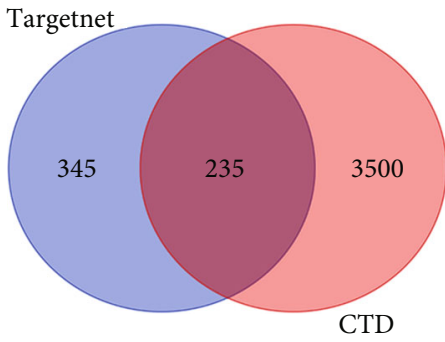

(b)

FIGURE 2: Resveratrol-related targets: (a) the green circle on the left represents targets from TargetNet, the blue circle on the right represents targets from CTD, and the yellow circle in the middle indicates the overlapping targets of TargetNet and CTD. (b) Targets from TargetNet and CTD are shown in the Venn diagram.

pharmacology methodology [9]. Resveratrol possesses not only antiviral properties but also anti-inflammatory effects; however, there is still no research focused on Resveratrol in the treatment of COVID-19 at the moment. In this study, a network pharmacological approach and bioinformatic gene analysis strategy were adopted to investigate the mechanism of action underlying the effectiveness of Resveratrol in COVID-19 therapy.

\section{Materials and Methods}

2.1. Potential Resveratrol-Related Targets. The word "Resveratrol" was searched in PubChem (https://pubchem.ncbi.nlm .nih.gov/), which is the world's largest collection of freely accessible chemical information [10]; molecular structure and PubChem CID (445154) of Resveratrol were obtained. Comparative Toxicogenomics Database (CTD, http:// 


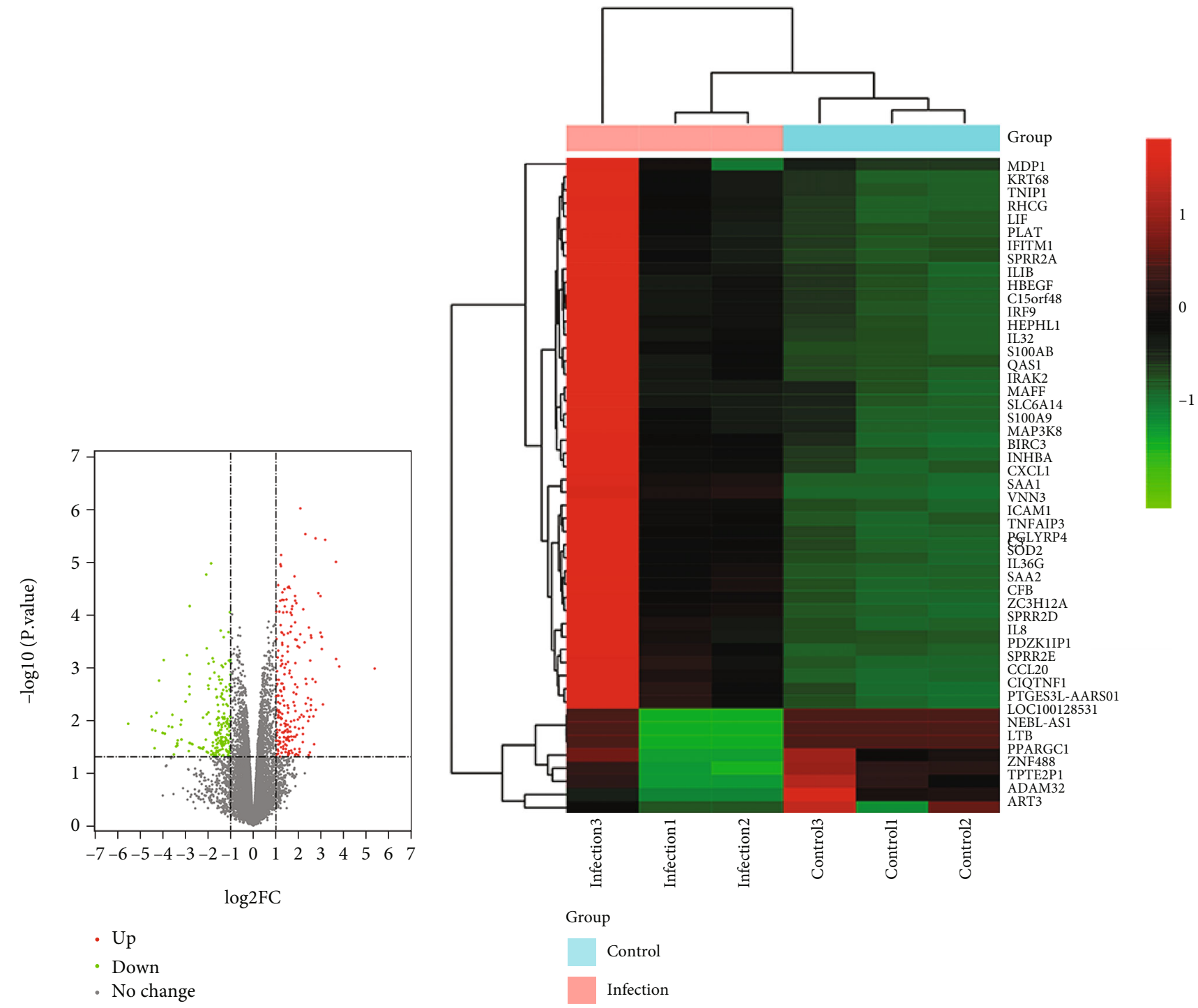

(a)

(b)

FIGURE 3: (a) Volcano plots of the significantly expressed ( $p<0.05$, fold change $>1)$ mRNAs between control and SARS-CoV-2 samples after analysis of the GSE147507 dataset. Red dots represent upregulated genes; green dots represent downregulated genes. (b) Heatmaps depicting the expression levels of the top 50 DEGs ranked by $p$ value among SARS-CoV-2 DEGs. Legend on the top right indicates log fold change of genes (Infection1, Infection2, Infection3 = infected with SARS-CoV-2 samples; Control1, Control2, Control3=mocktreated samples).

ctdbase.org/about/), a robust publicly available database that provides manually curated information about chemicalgene/protein interactions and chemical-disease and genedisease relationships [11], and TargetNet (http://targetnet .scbdd.com), an open web server that is used for netting or predicting the binding of multiple targets for any given molecule [12], were used to predict potential targets for Resveratrol. To improve accuracy, we took the target genes found in the intersecting region of these two databases. Because of the nonstandard naming, the names of targets were listed using the official symbol format from the UniProt Knowledgebase (UniProtKB, http://www.uniprot.org/).

2.2. SARS-CoV-2-Related Genes. The GSE147507 dataset containing the host transcriptional response to SARS-CoV-
2 was downloaded from GEO [13]. During the publisher's study, SARS-CoV-2 (USA-WA1/2020) was used to stimulate primary human lung epithelium (NHBE) and transformed lung alveolar (A549) cells, which suggested that the unique transcriptional signature of this virus may be responsible for the development of COVID-19 [14]. We chose the transcriptional results of NHBE for analysis. R packages of "impute" and "limma" provided by the Bioconductor project (http:// www.bioconductor.org/packages/release/bioc/html/affy.html) [15] were applied to assess the transcriptional results of NHBE. Quantile normalization and log2-transformation were used to create a robust multiarray average (RMA). Adjusted original $p$ values were obtained via the Benjamini-Hochberg method; the false discovery rate (FDR) procedure was used to calculate fold changes (FC). Gene expression values of $\mid \log$ 


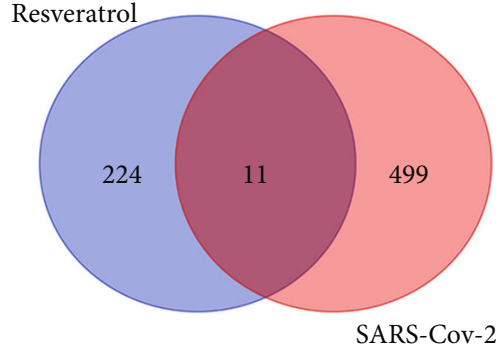

(a)

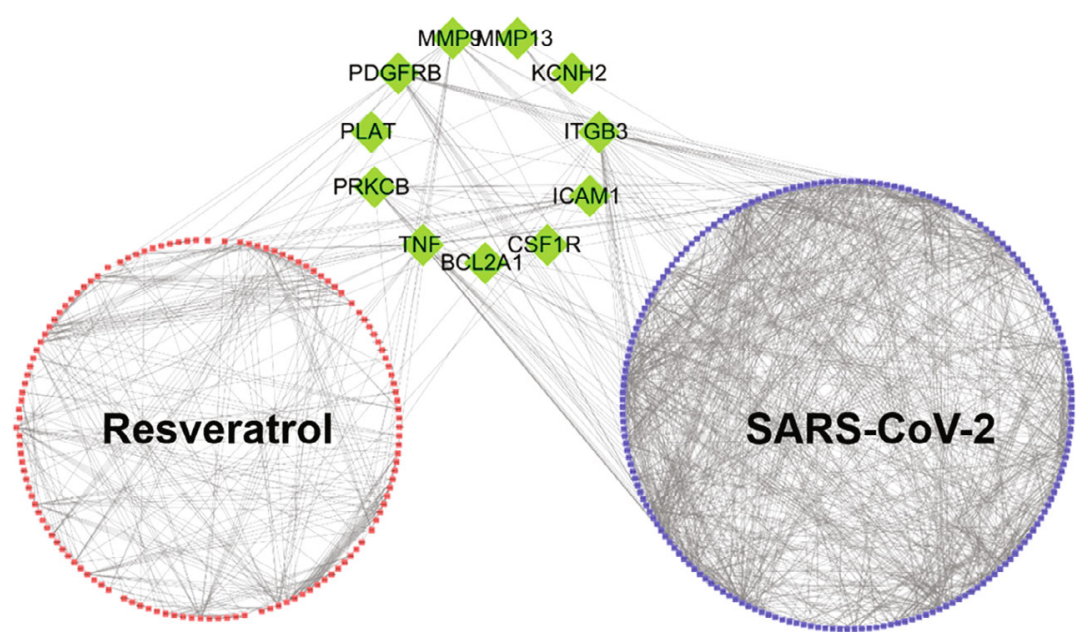

(b)

FIgure 4: PPI network analysis. (a) Targets of Resveratrol and SARS-CoV-2 DEGs are shown in the Venn diagram. There are 235 Resveratrol-related targets and 510 SARS-CoV-2 DEGs, and both share 11 targets. (b) The red circle on the left represents Resveratrolrelated targets, the blue circle on the right represents SARS-CoV-2 DEGs, and the green rhombus in the middle indicates the overlapping targets between Resveratrol and SARS-CoV-2 DEGs; edges represent correlations between targets.

$2 \mathrm{FC} \mid>1$ and $p$ value $<0.05$ were used as a threshold to filter differentially expressed genes (DEGs).

2.3. PPI Network Construction. Intersecting target genes of Resveratrol and DEGs related to SARS-CoV-2 were obtained. The Resveratrol-related targets and SARS-CoV-2 DEGs were uploaded to String (https://string-db.org/) [16], with species set as "Homo sapiens," a confidence score $>$ 0.9 to construct PPI networks, and then, the 2 PPI networks were merged and visualized using Cytoscape 3.7.2 (http:// www.cytoscape.org/) [17].

2.4. KEGG Pathway and Gene Ontology (GO) Enrichment Analysis. A list of Resveratrol targets and DEGs related to SARS-CoV-2 were submitted to Metascape (http:// metascape.org) [18], with species limited to "Homo sapiens." KEGG pathway, GO Biological Processes, GO Cellular Components, and GO Molecular Functions analysis were carried out with the following ontology sources: the enrichment background are all genes in the genome. $p$ value $<0.01$, count $>3$, and a minimum enrichment factor of 1.5 were used as filtering terms. Common KEGG pathways and GO terms between Resveratrol-related targets and SARS-CoV2's DEGs were chosen. We listed the overlapping targets and related key pathways. KEGG Mapper queried the location of SARS-CoV-2 DEGs and shared targets in the key pathways.

2.5. In Silico Molecular Docking Study of Resveratrol Key Targets. Molecular docking of Resveratrol with the common targets between Resveratrol and SARS-CoV-2 was performed using Autodock Vina [19]. The molecular structure of Resveratrol was downloaded in the PDB format from the PubChem database (https://www.ncbi.nlm.nih.gov/) [20]. The molecular structures of the targets were obtained from the Protein Data Bank (http://www.rcsb.org/) [21]. Before docking, the original crystal ligands and water mole- cules were removed from the protein-ligand complexes. Hydrogen atoms and charge were added, and default settings were selected for other parameters. Local Search Parameters were selected as the molecular docking model of Resveratrol to the protein targets. The docking score was used to evaluate the theoretical binding affinities of Resveratrol to the common targets.

\section{Results}

3.1. Potential Targets of Resveratrol. The molecular structure of Resveratrol was downloaded from the PubChem database (Figure 1). Then, 616 corresponding potential targets of Resveratrol were extracted from TargetNet, while 3735 corresponding potential targets of Resveratrol were obtained from the Comparative Toxicogenomics Database (CTD). After comparing common targets, 235 potential targets were selected, as shown in Figure 2 and Supplemental file Table S1.

3.2. Identification of SARS-CoV-2 DEGs. In total, we identified 23710 genes when comparing SARS-CoV-2 and control samples and 510 of them were considered significantly differentially expressed, including 270 downregulated genes and 240 upregulated genes. Heatmaps were used to display the expression levels of the top 50 DEGs ranked by $p$ value (Figure 3 and Supplemental file Table S2).

3.3. PPI Network Analysis. Overlapping targets of Resveratrol and SARS-CoV-2 DEGs included the following: MMP13, PRKCB, PLAT, KCNH2, ICAM1, PDGFRB, TNF, ITGB3, CSF1R, BCL2A1, and MMP9. PPI network was used to visualize and quantify the function of specific proteins in cells at the systematic level [22]. PPI network of Resveratrolrelated targets and SARS-CoV-2 DEGs were constructed, and the common targets were identified (Figure 4). 

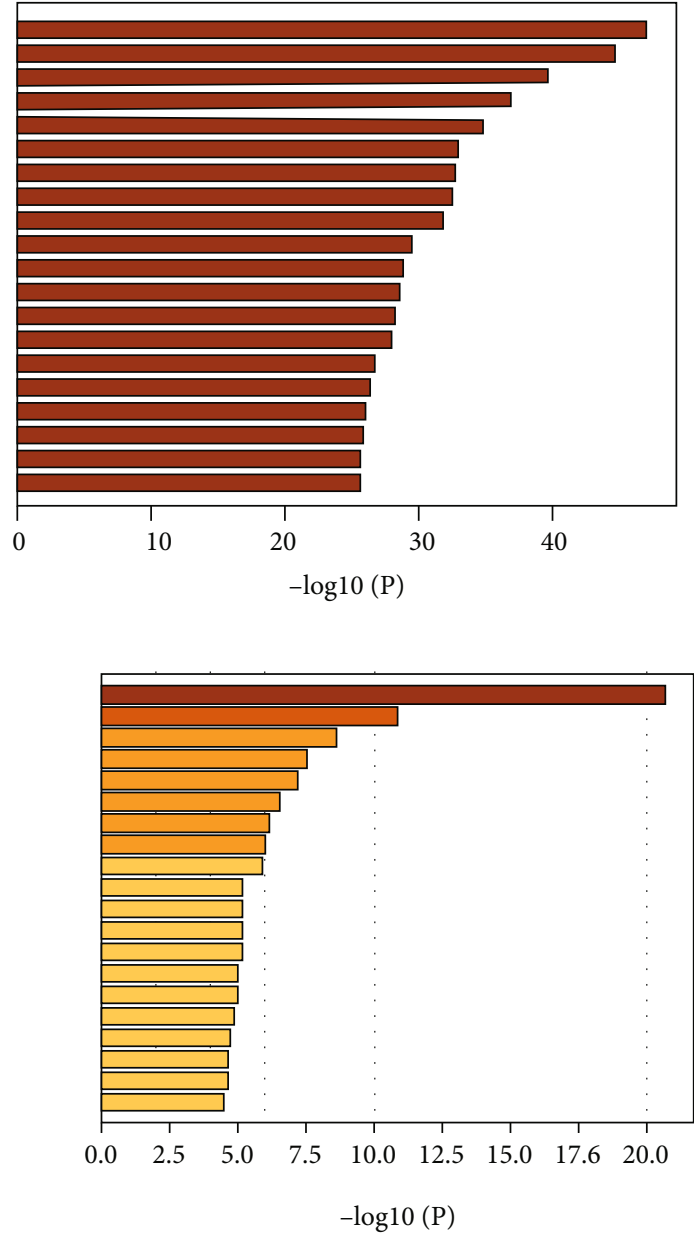

GO: 0004672:Protein kinase activity GO: 1901699:Cellular response to nitrogen compound GO: 0007169:Transmembranereceptor protein tyrosine kinase signaling pathway GO: 00071407:Cellular response to organic cyclic compound GO: 0009611:Response to wounding GO: 0003011:Circulatory system process GO: 0030335:Positive regulation of cell migration GO: 0051347:Positive regulation of transferase activity GO: 0048511:Rhythmic process GO: 0010035:Response to inorganic substance GO: 0010035:Response to molecule of bacterial origin GO: 0019901:Protein kinase binding GO: 0010942:Positive regulation of cell death GO: 0070997: Neuron death

GO: 0035690:Cellular response to drug GO: 004677:Protein autophosphorylation GO: 0097190:Apoptotic signaling pathway GO: 0030155:Regulation of cell adhesion GO: 0006935:Chemotaxis GO: 0019221:Cytokine-mediated signaling pathway (a)

GO:0019221: Cytokine-mediated signaling pathway GO:0032496: Response to lipopplysaccharide GO:0051607: Defense response to virus GO:0007159: Leukocyte cell-cell adhesion

GO:0032103: Positive regulation of response to external stimulus

GO:0050730: Regulation of peptidly-tyrosine phosphorylation

GO:0071356: Cellular response to tumor necrosis factor

GO:0002526: Acute inflammatory response

GO:0017159: Pantetheine hydrolase activity

GO:0019221: Protein kinase activity

GO:0043410: Positive regulation of MAPK cascade

GO:0052548: Regulation of endopeptidase activity

GO:0002274: Myeloid leukocyte activation

GO:2000147: Positive regulation of cell motility

GO:0070268: Cornification

GO:0002687: Positive regulation of leukocyte migration

GO:0045124: Regulation of bone resorption

GO:0030225: Macrophage differentiation

GO:0048661: Positive regulation of smooth muscle cell proliferation

GO:0030949: Positive regulation of vascular endothelial growth factor receptor signaling pathway GO:0001819: Positive regulation of cytokine production

(b)

FIGURE 5: GO enrichment analysis. (a) The most significant items in Resveratrol-related targets are classified according to the $p$ value. (b) The most significant items in SARS-CoV-2 DEGs are ranked by $p$ value.

TABlE 1: Top 10 overlapping GO terms between Resveratrolrelated targets and SARS-CoV-2 DEGs ranked by $p$ value.

\begin{tabular}{lcc}
\hline Term & Description & $p$ value \\
\hline GO:0019221 & $\begin{array}{c}\text { Cytokine-mediated signaling } \\
\text { pathway }\end{array}$ & $2.05074 E-21$ \\
GO:0060326 & Cell chemotaxis & $5.87962 E-12$ \\
GO:0030595 & Leukocyte chemotaxis & $7.40744 E-12$ \\
GO:0032496 & Response to lipopolysaccharide & $1.40404 E-11$ \\
GO:0006935 & $\begin{array}{c}\text { Chemotaxis } \\
\text { GO:0097529 }\end{array}$ & $\begin{array}{c}\text { Myeloid leukocyte migration } \\
\text { GO:0071222 }\end{array}$ \\
Cellular response to \\
GO:0042330 & $\begin{array}{c}\text { lipopolysaccharide } \\
\text { Taxis }\end{array}$ & $1.7267 E-11$ \\
GO:0002237 & $\begin{array}{c}\text { Response to molecule of bacterial } \\
\text { origin }\end{array}$ & $5.64373 E-11$ \\
GO:0071219 & Cellular response to molecule of & $5.83252 E-11$ \\
\hline & bacterial origin & $1.85514 E-11$ \\
\hline
\end{tabular}

3.4. Gene Ontology and KEGG Enrichment Analysis of Resveratrol-Related Targets and SARS-CoV-2 DEGs. GO analysis identified 266 enriched terms for Resveratrolrelated targets, including 240 Biological Process terms, 23 Molecular Function terms, and 3 Cellular Component terms. The most significant items ranked by $p$ value are listed in Figure 5(a) and Supplemental file Table S3. SARS-CoV-2 DEGs displayed 315 enriched GO terms, including 299 Biological Process terms, 13 Molecular Function terms, and 3 Cellular Component terms. The most significant items were ranked using $p$ value and are listed in Figure 5(b) and Supplemental file Table S4. The intersecting region between Resveratrol-related targets and SARS-CoV-2 DEGs contained 89 terms; the top 10 common items were ranked by $p$ value and are listed in Table 1.

For Resveratrol-related targets, KEGG analysis revealed 142 enriched pathways and the most significant pathways were listed by $p$ value strength in Figure 6(a) and Supplemental file Table S5. For SARS-CoV-2 DEGs, KEGG analysis showed 40 enriched pathways, ranked by $p$ value in Figure 6(b) and Supplemental file Table S6. The intersection of Resveratrol-related targets and SARS-CoV-2 DEGs included 30 terms, the top 10 items in the intersection 


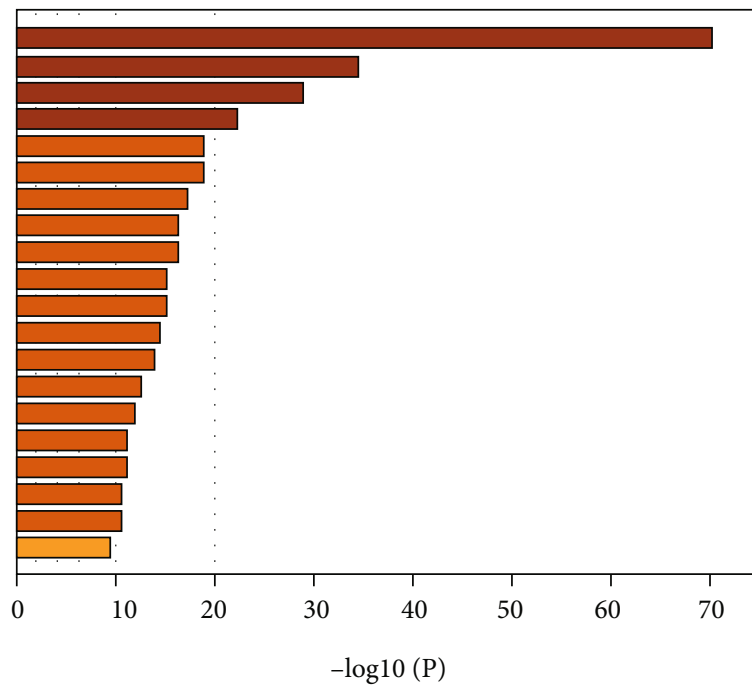

hsa05200: Pathways in cancer

hsa04933: AGE-RAGE signaling pathway in diabetic complications hsa04071: Sphingolipid signaling pathway

hsa05206: Micro RNA in cancer

hsa05203: Viral carcinogenesis

hsa05203: Neuroactive ligand-receptor interaction

hsa04152: AMPK singnaling pathway

hsa04657: IL-17 signaling pathway

hsa04726: Serotonergic synapse

hsa04064: NF-kappa B signaling pathway

hsa04670: Leukocyte transendothelial migration

hsa05219: Bladder cancer

hsa05202: Transcriptional misregulation in cancer

hsa04650: Natural killer cell mediated cytotoxicity

hsa05010: Alzheimer's disease

hsa04520: Adherens junction

hsa04750: Inflammatory mediator regulation of TRP channels

hsa04215: Apoptosis-multiple specie

hsa04728: Dopaminergic synapse

hsa00590: Arachidonic acid metabolism

(a)

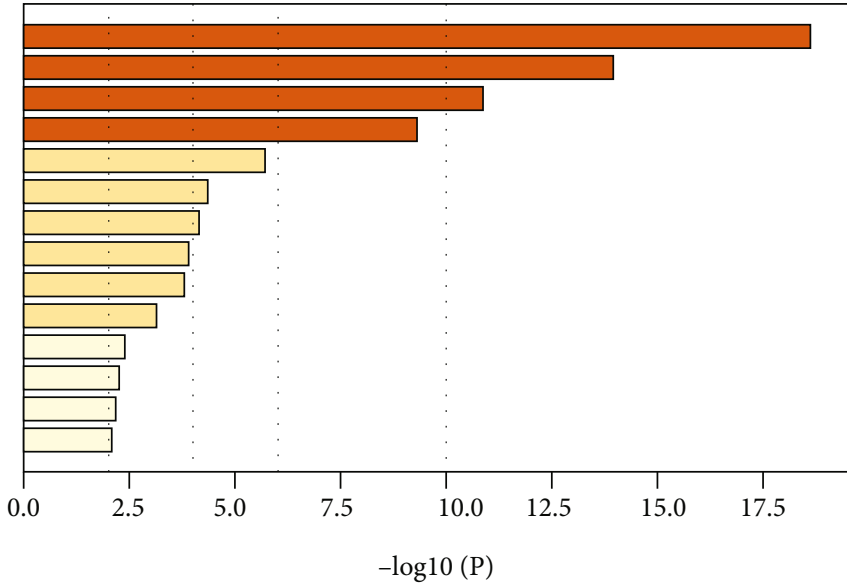

hsa04060: Cytokine-cytokine receptor interaction hsa04657: IL-17 signaling pathway

hsa05323: Rheumatiod artheits

hsa04621: NOD-like receptor signaling pathway

hsa04064: NF_kappa B signaling pathway

hsa04630: Jak-STAT signaling pathway

hsa05418: Fluid shear stress and atherosclerosis

hsa04610: Complement and coagulation cascades

hsa05202: Transcriptional misregulation in cancer

hsa04015: Rap1 signaling patheway

hsa04931: Insulin resistance

hsa04670: Leukocyte transendothelial migration

hsa05219: Bladder cancer

hsa04664: Fc epsilon RI signaling pathway

(b)

FIGURE 6: KEGG enrichment analysis. (a) The most significant enriched KEGG pathways are classified according to their $p$ value in Resveratrol-related targets. (b) The most significant enriched KEGG pathways are ranked by $p$ value in SARS-CoV-2 DEGs.

TABle 2: Top 10 overlapping KEGG pathways between Resveratrol-related targets and SARS-CoV-2 DEGs ranked by $p$ value.

\begin{tabular}{lcc}
\hline Term & Description & $p$ value \\
\hline hsa04657 & IL-17 signaling pathway & $9.59366 E-15$ \\
hsa04668 & TNF signaling pathway & $1.69723 E-13$ \\
hsa04621 & NOD-like receptor signaling pathway & $6.07599 E-10$ \\
hsa05162 & Measles & $5.30173 E-09$ \\
hsa05134 & Legionellosis & $5.45103 E-09$ \\
hsa05164 & Influenza A & $2.00359 E-07$ \\
hsa05133 & Pertussis & $1.63392 E-06$ \\
hsa04064 & NF-kappa B signaling pathway & $1.8066 E-06$ \\
hsa05140 & Leishmaniasis & $9.37339 E-06$ \\
hsa05146 & Amoebiasis & $1.38036 E-05$ \\
\hline
\end{tabular}

ordered by $p$ value are listed in Table 2 . The common targets and overlapping KEGG pathways are listed in Figure 7. The key KEGG pathways and the location of SARS-CoV-2 DEGs and overlapping genes of enriched pathways are listed in Figure 8.

3.5. Molecular Docking Analysis. Molecular docking analysis showed that the docking scores of Resveratrol in relation to PLAT, MMP13, PRKCB, ICAM1, and ITGB3 are greater than $5 \mathrm{kcal} / \mathrm{mol}$. Interestingly, Resveratrol displayed the highest docking score with PLAT and MMP13 (docking score: $-6.93,-6.27)$, demonstrating that Resveratrol could form a strong interaction with PLAT and MMP13. Other key targets that showed an affinity with Resveratrol are further listed in Figure 9 and Table 3.

\section{Discussion}

We screened 235 potential Resveratrol-related targets from an online database; GO analysis of these targets revealed 


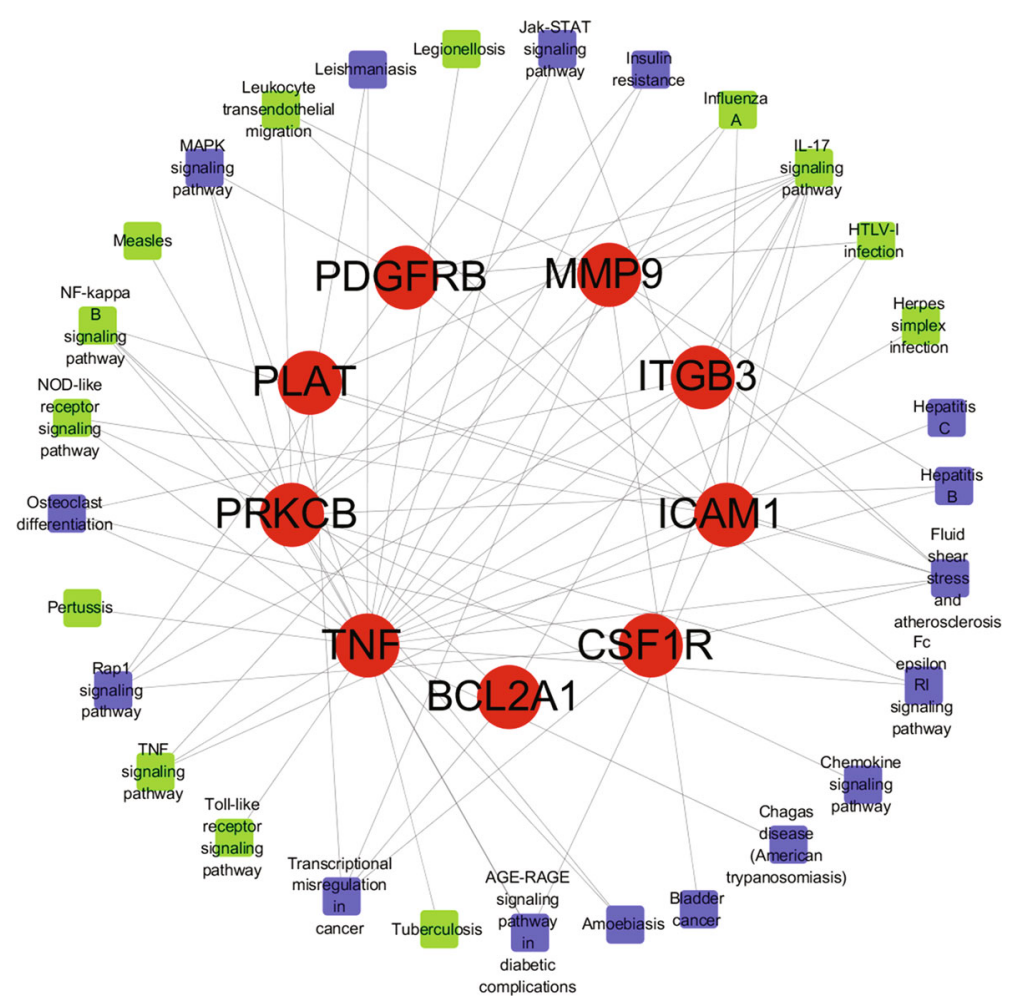

Figure 7: The shared targets have overlapping KEGG pathways. The red circle in the middle represents overlapping Resveratrol-related targets and SARS-CoV-2 DEGs; the green square represents overlapping KEGG pathways in relation to inflammatory and immune response; the blue square represents other items among overlapping KEGG pathways.

enriched terms such as cytokine-mediated signaling pathway, cell chemotaxis, leukocyte chemotaxis, response to lipopolysaccharide, chemotaxis, myeloid leukocyte migration, and cellular response to lipopolysaccharide, which are in line with the findings of a recent study on Resveratrol [23]. 510 DEGs were then confirmed in samples infected with SARSCoV-2 when compared to mock samples. Similar to the study by Blanco-Melo et al. [24], enriched GO terms such as cellular response to virus infection (GO:0009615), humoral immune response (GO:0006959), and chemokines and cytokines (GO:0005125) were identified.

Among the SARS-CoV-2 DEGs identified, cytokines such as IL-6, IL-8, IL-17, TNF, IL-32, IL-1, and NOD2 were upregulated. These results are in accordance with a recent study that found elevated levels of cytokines in the plasma of COVID-19 patients [1]. GO analysis results of SARSCoV-2 DEGs reveal that cytokine-mediated signaling pathway, cytokine activity, and neutrophil chemotaxis are the most significant terms. The most relevant KEGG enrichment items were cytokine-cytokine receptor interaction, IL-17 signaling pathway, TNF signaling pathway, NODlike receptor signaling pathway, and NF- $\kappa \mathrm{B}$ signaling pathway. Recent research shows that the NF- $\kappa$ B pathway can be induced by SARS-CoV-2 infection, leading to multiple inflammatory responses [25]. In addition, TNF was present in the blood and disease tissues of patients with COVID-19 [26], which is important in nearly all acute inflammatory reactions, acting as an amplifier of inflammation. Furthermore, $N F-\kappa B, I L-6$, and $T N F$ are considered promising therapeutic targets in COVID-19 [27].
The shared targets of Resveratrol and SARS-CoV-2 DEGs may represent the potential therapeutic targets of Resveratrol on COVID-19, which include $M M P 13, P R K C B$, PLAT, KCNH2, ICAM1, PDGFRB, TNF, ITGB3, CSF1R, $B C L 2 A 1$, and MMP9. These are mainly involved in the IL17 signaling pathway, NF- $\kappa$ B signaling pathway, and TNF signaling pathway. The activation of these pathways leads to the increased release of cytokines, which have been shown to play an important role in viral infection [28, 29]. We surmise that Resveratrol can reduce the expression level of cytokines and alleviate hyperinflammation in COV19 by inhibiting the activation of these pathways.

Studies have demonstrated that Resveratrol exerts antiinflammatory effects through various pathways to reduce lung injury [30]. For instance, Resveratrol suppresses TNFinduced activation of nuclear transcription factors NF- $\kappa \mathrm{B}$ [31] and also mitigates LPS-induced acute lung inflammation by inhibiting the TLR4/NF- $\kappa$ Bp $65 / \mathrm{MAPK}$ signaling cascade and NLRP3 inflammasome [32-34]. Furthermore, Resveratrol improved hyperoxia-induced lung injury via its antioxidant, anti-inflammatory, and antifibrotic effects, promoting the transdifferentiation of alveolar type II epithelial cells into their type I counterpart and suppressing the $\mathrm{Wnt} / \beta$-catenin signaling in preterm rats $[35,36]$.

By decreasing nucleocapsid $(\mathrm{N})$ protein expression, Resveratrol controlled MERS-CoV infection and improved cellular survival after virus infection [37]. The interaction of nucleocapsid protein and viral RNA in the cytoplasm is necessary for viral RNA nucleocapsid assembly. Resveratrol inhibited the replication of influenza A by nearly $90 \%$ by 


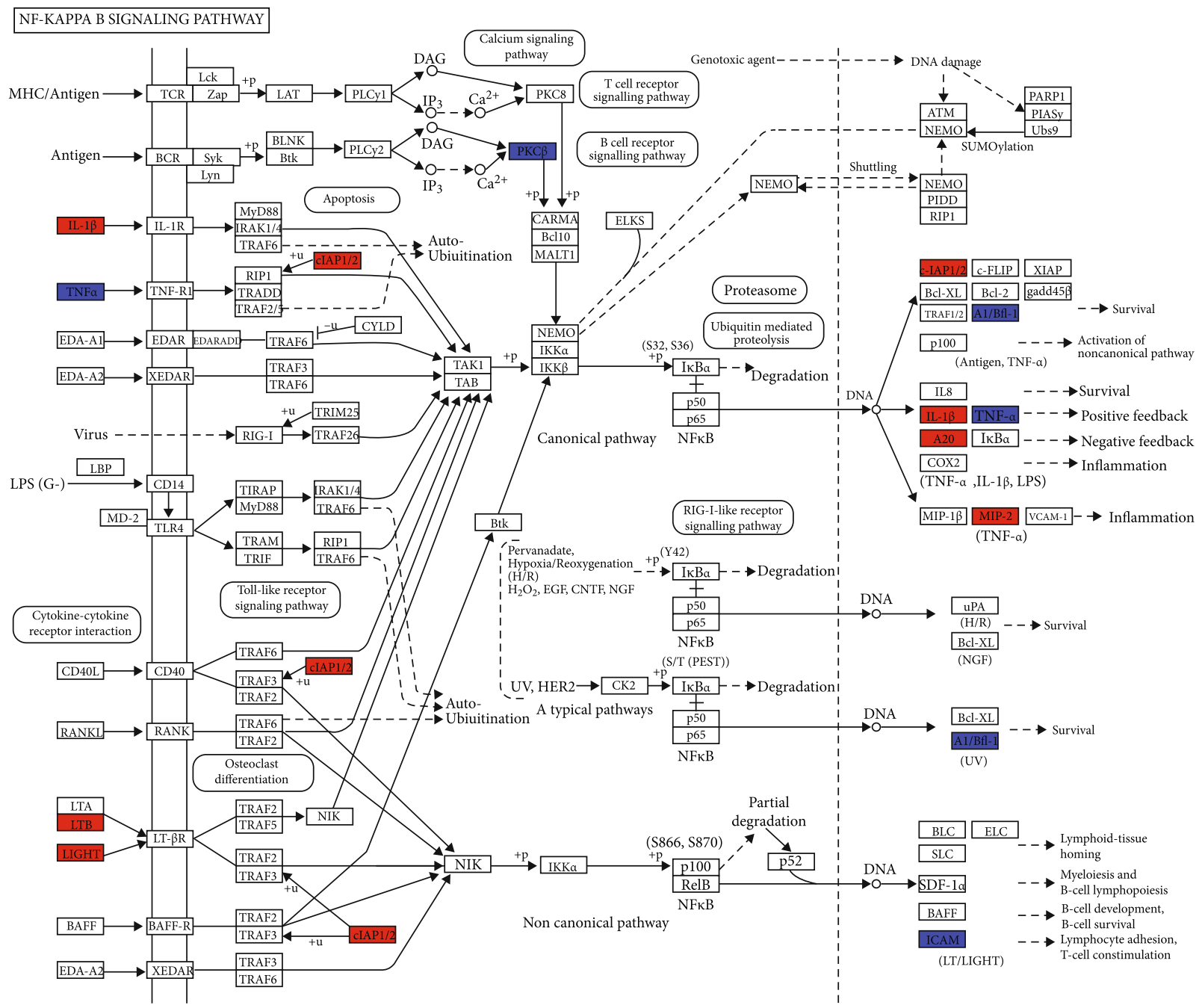

(a)

Figure 8: Continued. 


\section{INF SIGNALING PATHWAY}

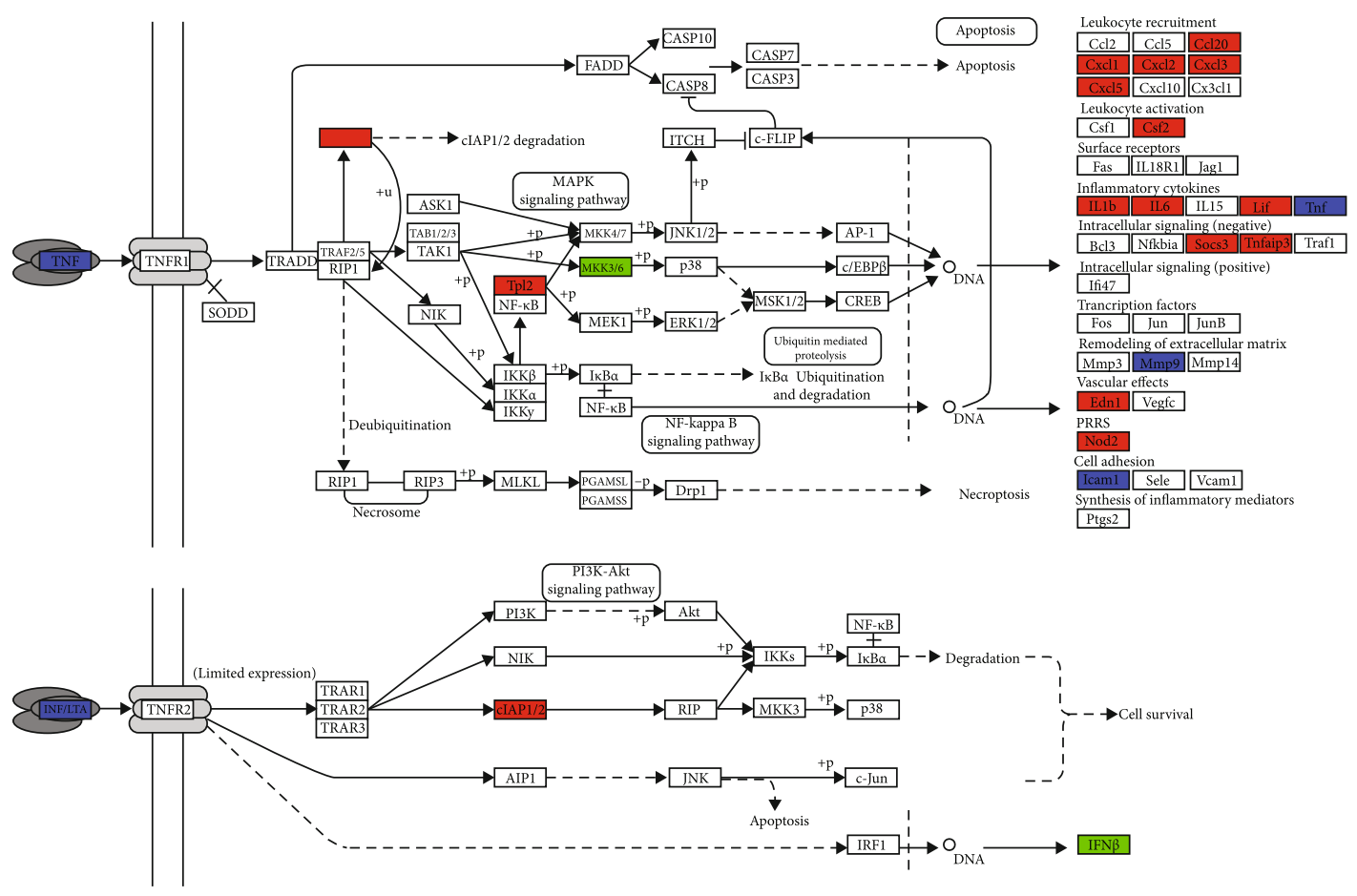

(b)

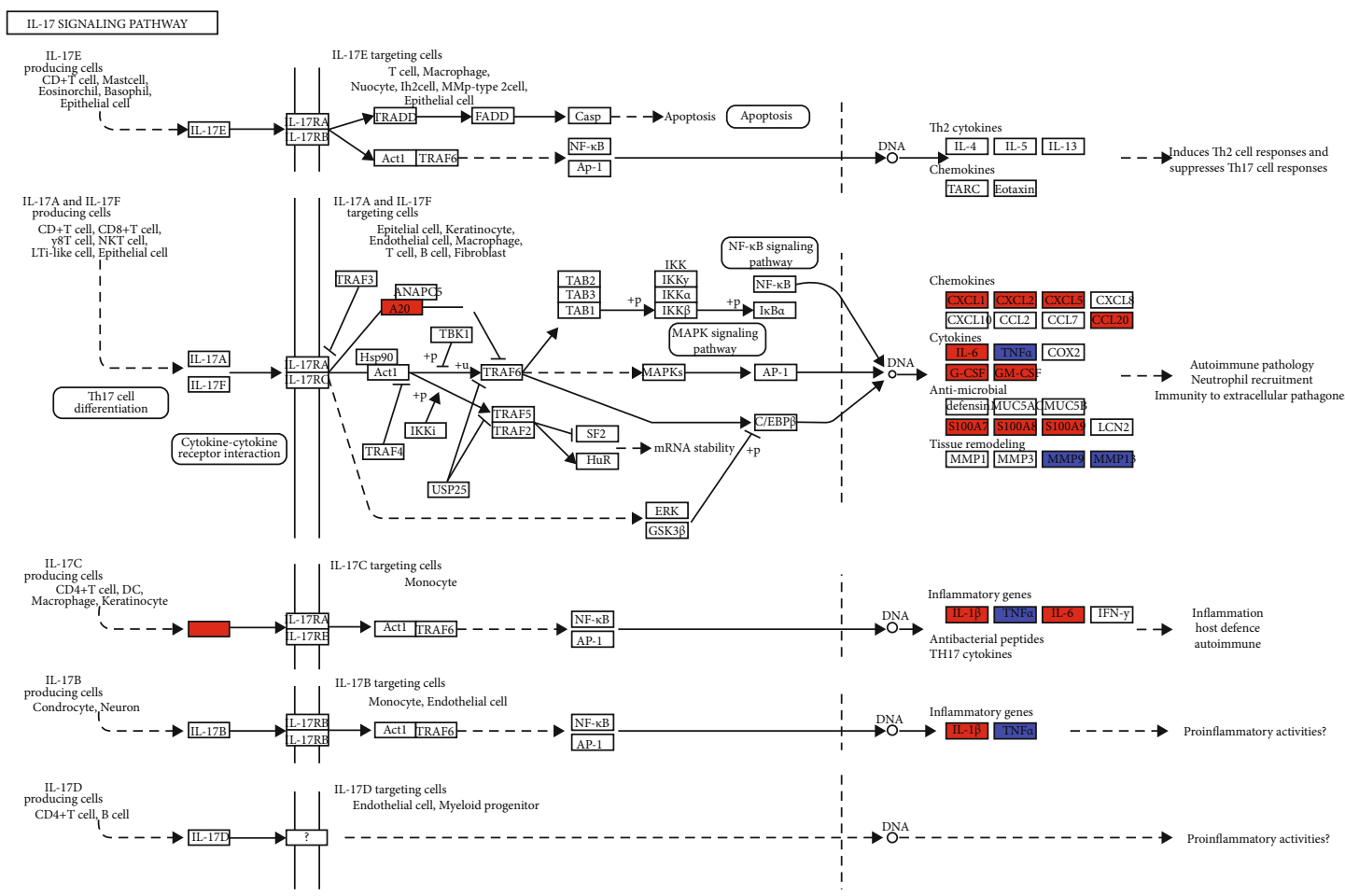

(c)

FIGURE 8: The key KEGG pathways: (a) NF- $\kappa$ B signaling pathway, (b) TNF signaling pathway, and (c) IL-17 signaling pathway. The red nodes represent upregulated SARS-CoV-2 DEGs, the green marked node represents downexpression SARS-CoV-2 DEGs, and the blue marked node represents overlapping targets between Resveratrol and SARS-CoV-2. 

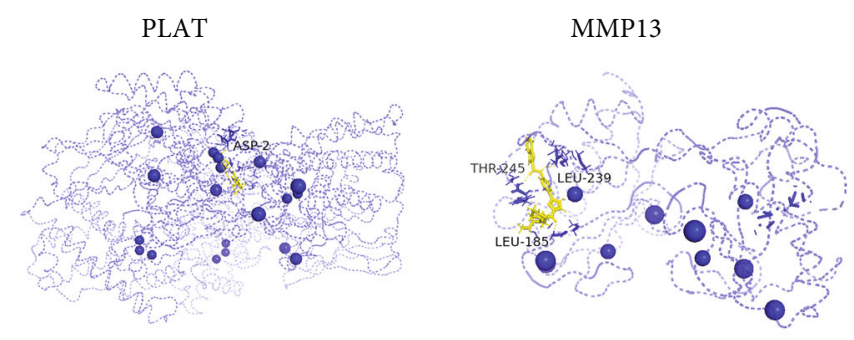

PRKCB
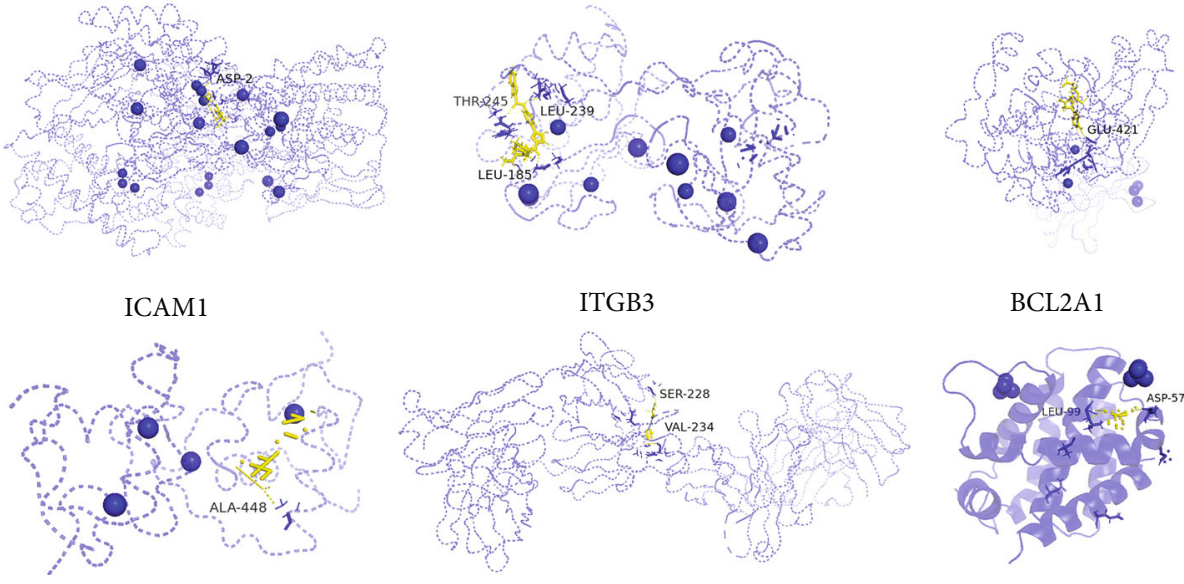

BCL2A1

Figure 9: Structural interactions of Resveratrol and key target receptors.

TABLE 3: The docking scores of Resveratrol and rapamycin with key proteins.

\begin{tabular}{lccc}
\hline Target & PDB ID & Drug & Binding energy $(\mathrm{kcal} / \mathrm{mol})$ \\
\hline PLAT & 1OLP & & -6.93 \\
MMP13 & 2OW9 & & -6.27 \\
PRKCB & 3PFQ & Resveratrol & -6.17 \\
ICAM1 & 5E6D & & -5.88 \\
ITGB3 & 4YNY & & -5.2 \\
BCL2A1 & 5UUP & & -4.98 \\
\hline
\end{tabular}

preventing nucleocapsid protein translocation from the nucleus to the cytoplasm [38, 39]. Resveratrol not only reduced the titer of the respiratory syncytial virus but also decreased interferon- $\gamma$ production induced by the respiratory syncytial virus in a mouse model and alleviated airway inflammation and hyperresponsiveness [23, 40]. In human 9HTEo cells, respiratory syncytial virus replication and production of IL-6 were reduced after Resveratrol incubation. Resveratrol treatment also decreased expression of ICAM-1 induced by human rhinoviruses in H1HeLa and nasal epithelial cells $[41,42]$. These data demonstrate that Resveratrol might act as a therapeutic drug for viral infections showing both effective anti-inflammatory and antiviral potential. Although there are no experiments to confirm the therapeutic effect of Resveratrol in COVID-19, the molecular docking previously revealed a wide spectrum of interactions between Resveratrol derivatives and two newly released coordinate structures for COVID-19 [43]. The drug for immunosuppression is likely to be beneficial to patients with hyperinflammation. IL-1 blockade (anakinra) showed a significant survival benefit in sepsis patients with hyperinflammation, without increased adverse events [44].

However, our study has some limitations. We failed to prove the therapeutic effect of Resveratrol in COVID-19 through experiments due to complexity reasons. Direct evidence of Resveratrol efficacy is still needed in a SARS-CoV-2 infection experiment model. Nevertheless, we strongly believe that Resveratrol is likely to be beneficial for COVID-19 patients because combined anti-inflammatory and antiviral effects are shown in numerous studies. In light of our findings, related and more in-depth studies on Resveratrol are urgently warranted.

\section{Conclusion}

Taken together, our findings show that Resveratrol is a potential candidate in COVID-19 therapy, based on network pharmacology and bioinformatic gene analysis. More importantly, we also identified the possible key targets and pathways involved in this novel therapeutic strategy.
Abbreviations
COVID-19: Coronavirus disease 2019
CTD: Comparative Toxicogenomics Database
DEGs: Differentially expressed genes
GEO: Gene Expression Omnibus
GO: $\quad$ Gene Ontology
PPI: $\quad$ Protein-protein interaction
NF- $\kappa$ B: $\quad$ NF-kappa B
ARDS: $\quad$ Severe acute respiratory distress syndrome
NHBE: $\quad$ Primary human lung epithelium
A549: $\quad$ Transformed lung alveolar.

\section{Data Availability}

The data used in this study were obtained from open public databases, and data acquisition is explained in the manuscript. SARS-CoV-2 differentially expressed genes (DEGs) were taken from Gene Expression Omnibus (GEO) datasets GSE147507. The molecular structure of Resveratrol was downloaded from the PubChem database (https://pubchem .ncbi.nlm.nih.gov/). TargetNet (http://targetnet.scbdd.com/) was used to predict potential targets for Resveratrol. The molecular structures of the targets were obtained from the Protein Data Bank (http://www.rcsb.org/). 


\section{Conflicts of Interest}

The authors disclose no conflict of interest.

\section{Authors' Contributions}

Zijian Xiao, Qing Ye, Xiaomei Duan, and Tao Xiang contributed to this work. Qing Ye and Zijian Xiao participated in the design of this study, and Xiaomei Duan contributed to the statistical analysis. Tao Xiang drafted the manuscript. All authors have read and approved the final manuscript. Zijian Xiao and Qing Ye contributed equally to this work.

\section{Acknowledgments}

We thank all the doctors, nurses, and researchers who have fought against the virus on the frontline of the 2019-nCoV epidemic. We thank everyone who has given great and selfless support to the fight against this deadly infection.

\section{Supplementary Materials}

Supplementary 1. Supplementary Table S1: resveratrolrelated targets.

Supplementary 2. Supplementary Table S2: SARS-CoV-2 DEGs.

Supplementary 3. Supplementary Table S3: resveratrolrelated targets $\mathrm{GO}$ analysis-enriched terms.

Supplementary 4. Supplementary Table S4: SARS-CoV-2 DEGs GO analysis-enriched terms.

Supplementary 5. Supplementary Table S5: resveratrolrelated targets KEGG-enriched terms.

Supplementary 6. Supplementary Table S6: SARS-CoV-2 DEGs KEGG-enriched terms.

\section{References}

[1] C. Huang, Y. Wang, X. Li et al., "Clinical features of patients infected with 2019 novel coronavirus in Wuhan, China," The Lancet, vol. 395, no. 10223, pp. 497-506, 2020.

[2] M. D. Howell and A. M. Davis, "Management of ARDS in adults," JAMA, vol. 319, no. 7, pp. 711-712, 2018.

[3] X.-d. Zhu, L. X-p, and W.-b. Dong, "Resveratrol as a potential therapeutic drug for respiratory system diseases," Drug Design, Development and Therapy, vol. 11, pp. 3591-3598, 2017.

[4] U. Svajger and M. Jeras, "Anti-inflammatory effects of resveratrol and its potential use in therapy of immune-mediated diseases," International Reviews of Immunology, vol. 31, no. 3, pp. 202-222, 2012.

[5] L. Malaguarnera, "Influence of resveratrol on the immune response," Nutrients, vol. 11, no. 5, p. 946, 2019.

[6] N. Bostanghadiri, A. Pormohammad, A. S. Chirani, R. Pouriran, S. Erfanimanesh, and A. Hashemi, "Comprehensive review on the antimicrobial potency of the plant polyphenol resveratrol," Biomedicine \& Pharmacotherapy, vol. 95, pp. 1588-1595, 2017.

[7] V. Berardi, F. Ricci, M. Castelli, G. Galati, and G. Risuleo, "Resveratrol exhibits a strong cytotoxic activity in cultured cells and has an antiviral action against polyomavirus: potential clinical use," Journal of Experimental \& Clinical Cancer Research, vol. 28, no. 1, p. 96, 2009.

[8] C. N. Chan, B. Trinite, and D. N. Levy, "Potent inhibition of HIV-1 replication in resting CD4 T cells by resveratrol and pterostilbene," Antimicrobial Agents and Chemotherapy, vol. 61, no. 9, 2017.

[9] C. Ge and Y. He, "In silico prediction of molecular targets of astragaloside IV for alleviation of COVID-19 hyperinflammation by systems network pharmacology and bioinformatic gene expression analysis," Frontiers in Pharmacology, vol. 11, 556984 pages, 2020.

[10] X. Wang, Y. Shen, S. Wang et al., "PharmMapper 2017 update: a web server for potential drug target identification with a comprehensive target pharmacophore database," Nucleic Acids Research, vol. 45, no. W1, pp. W356-W360, 2017.

[11] A. P. Davis, C. J. Grondin, R. J. Johnson et al., "The Comparative Toxicogenomics Database: update 2019," Nucleic Acids Research, vol. 47, no. D1, pp. D948-D954, 2019.

[12] Z. J. Yao, J. Dong, Y. J. Che et al., "TargetNet: a web service for predicting potential drug-target interaction profiling via multi-target SAR models," Journal of Computer-Aided Molecular Design, vol. 30, no. 5, pp. 413-424, 2016.

[13] T. Barrett, S. E. Wilhite, P. Ledoux et al., "NCBI GEO: archive for functional genomics data sets-update," Nucleic Acids Research, vol. 41, pp. D991-D995, 2013.

[14] D. Blanco-Melo, B. E. Nilsson-Payant, W.-C. Liu et al., "Imbalanced host response to SARS-CoV-2 drives development of COVID-19," Cell, vol. 181, no. 5, pp. 1036-1045.e9, 2020.

[15] Z. Chen, M. McGee, Q. Liu, and R. H. Scheuermann, "A distribution free summarization method for Affymetrix GeneChip arrays," Bioinformatics, vol. 23, no. 3, pp. 321-327, 2007.

[16] D. Szklarczyk, A. L. Gable, D. Lyon et al., "STRING v11: protein-protein association networks with increased coverage, supporting functional discovery in genome-wide experimental datasets," Nucleic Acids Research, vol. 47, no. D1, pp. D607D613, 2019.

[17] D. Otasek, J. H. Morris, J. Bouças, A. R. Pico, and B. A.-O. Demchak, "Cytoscape automation: empowering workflowbased network analysis," Genome Biology, vol. 20, no. 1, p. ???, 2019.

[18] Y. Zhou, B. Zhou, L. Pache et al., "Metascape provides a biologist-oriented resource for the analysis of systems-level datasets," Nature Communications, vol. 10, no. 1, article $1523,2019$.

[19] O. Trott and A. J. Olson, "AutoDock Vina: improving the speed and accuracy of docking with a new scoring function, efficient optimization, and multithreading," Journal of Computational Chemistry, vol. 31, no. 2, pp. 455-461, 2010.

[20] S. Kim, J. Chen, T. Cheng et al., "PubChem in 2021: new data content and improved web interfaces," Nucleic Acids Research, vol. 49, no. D1, pp. D1388-D1395, 2021.

[21] H. Berman, K. Henrick, and H. Nakamura, "Announcing the worldwide Protein Data Bank," Nature Structural \& Molecular Biology, vol. 10, no. 12, pp. 980-980, 2003.

[22] F. Jordan, T. P. Nguyen, and W. C. Liu, "Studying proteinprotein interaction networks: a systems view on diseases," Briefings in Functional Genomics, vol. 11, no. 6, pp. 497-504, 2012.

[23] N. Zang, S. Li, W. Li et al., "Resveratrol suppresses persistent airway inflammation and hyperresponsivess might partially 
via nerve growth factor in respiratory syncytial virus- infected mice," International Immunopharmacology, vol. 28, no. 1, pp. 121-128, 2015.

[24] D. Blanco-Melo, B. E. Nilsson-Payant, W.-C. Liu et al., "SARS$\mathrm{CoV}-2$ launches a unique transcriptional signature from in vitro, ex vivo, and in vivo systems," 2020.

[25] M. Murakami, D. Kamimura, and T. Hirano, "Pleiotropy and specificity: insights from the interleukin 6 family of cytokines," Immunity, vol. 50, no. 4, pp. 812-831, 2019.

[26] L. Wang, W. He, X. Yu et al., "Coronavirus disease 2019 in elderly patients: characteristics and prognostic factors based on 4-week follow-up," The Journal of Infection, vol. 80, no. 6 , pp. 639-645, 2020.

[27] P. Mehta, D. F. McAuley, M. Brown et al., "COVID-19: consider cytokine storm syndromes and immunosuppression," The Lancet, vol. 395, no. 10229, pp. 1033-1034, 2020.

[28] T. Kawai and S. Akira, "Innate immune recognition of viral infection," Nature Immunology, vol. 7, no. 2, pp. 131-137, 2006.

[29] T. Kawai and S. Akira, "Antiviral signaling through pattern recognition receptors," Journal of Biochemistry, vol. 141, no. 2, pp. 137-145, 2007.

[30] S. A. Rieder, P. Nagarkatti, and M. Nagarkatti, "Multiple antiinflammatory pathways triggered by resveratrol lead to amelioration of staphylococcal enterotoxin B-induced lung injury," British Journal of Pharmacology, vol. 167, no. 6, pp. 1244-1258, 2012.

[31] S. K. Manna, A. Mukhopadhyay, and B. B. Aggarwal, "Resveratrol suppresses TNF-induced activation of nuclear transcription factors NF- $\kappa \mathrm{B}$, activator protein-1, and apoptosis: potential role of reactive oxygen intermediates and lipid peroxidation," The Journal of Immunology, vol. 164, no. 12, pp. 6509-6519, 2000.

[32] G. Wang, Z. Hu, Q. Fu et al., "Resveratrol mitigates lipopolysaccharide-mediated acute inflammation in rats by inhibiting the TLR4/NF- $\kappa$ Bp65/MAPKs signaling cascade," Scientific Reports, vol. 7, no. 1, p. 45006, 2017.

[33] L. Jiang, L. Zhang, K. Kang et al., "Resveratrol ameliorates LPSinduced acute lung injury via NLRP3 inflammasome modulation," Biomedicine \& Pharmacotherapy, vol. 84, pp. 130-138, 2016.

[34] L. Ma, Y. Zhao, R. Wang et al., "3,5,4'-Tri-O-acetylresveratrol attenuates lipopolysaccharide-induced acute respiratory distress syndrome via MAPK/SIRT1 pathway," Mediators of Inflammation, vol. 2015, Article ID 143074, 12 pages, 2015.

[35] W. Xu, Y. Zhao, B. Zhang et al., "Resveratrol attenuates hyperoxia-induced oxidative stress, inflammation and fibrosis and suppresses $\mathrm{Wnt} / \beta$-catenin signalling in lungs of neonatal rats," Clinical and Experimental Pharmacology \& Physiology, vol. 42, no. 10, pp. 1075-1083, 2015.

[36] P. L. Liu, I. W. Chong, Y. C. Lee et al., "Anti-inflammatory effects of resveratrol on hypoxia/reoxygenation-induced alveolar epithelial cell dysfunction," Journal of Agricultural and Food Chemistry, vol. 63, no. 43, pp. 9480-9487, 2015.

[37] S. C. Lin, C. T. Ho, W. H. Chuo, S. Li, T. T. Wang, and C. C. Lin, "Effective inhibition of MERS-CoV infection by resveratrol," BMC Infectious Diseases, vol. 17, no. 1, p. 144, 2017.

[38] A. T. Palamara, L. Nencioni, K. Aquilano et al., "Inhibition of influenza A virus replication by resveratrol," The Journal of Infectious Diseases, vol. 191, no. 10, pp. 1719-1729, 2005.
[39] L. L. Newcomb, R. L. Kuo, Q. Ye, Y. Jiang, Y. J. Tao, and R. M. Krug, "Interaction of the influenza a virus nucleocapsid protein with the viral RNA polymerase potentiates unprimed viral RNA replication," Journal of Virology, vol. 83, no. 1, pp. 29-36, 2009.

[40] N. Zang, X. Xie, Y. Deng et al., "Resveratrol-mediated gamma interferon reduction prevents airway inflammation and airway hyperresponsiveness in respiratory syncytial virus-infected immunocompromised mice," Journal of Virology, vol. 85, no. 24, pp. 13061-13068, 2011.

[41] X. H. Xie, N. Zang, S. M. Li et al., "Resveratrol inhibits respiratory syncytial virus-induced IL-6 production, decreases viral replication, and downregulates TRIF expression in airway epithelial cells," Inflammation, vol. 35, no. 4, pp. 1392-1401, 2012.

[42] P. Mastromarino, D. Capobianco, F. Cannata et al., "Resveratrol inhibits rhinovirus replication and expression of inflammatory mediators in nasal epithelia," Antiviral Research, vol. 123, pp. 15-21, 2015.

[43] A. Ranjbar, M. Jamshidi, and S. Torabi, "Molecular modelling of the antiviral action of resveratrol derivatives against the activity of two novel SARS CoV-2 and 2019-nCoV receptors," European Review for Medical and Pharmacological Sciences, vol. 24, no. 14, pp. 7834-7844, 2020.

[44] B. Shakoory, J. A. Carcillo, W. W. Chatham et al., "Interleukin1 receptor blockade is associated with reduced mortality in sepsis patients with features of macrophage activation syndrome: reanalysis of a prior phase III trial," Critical Care Medicine, vol. 44, no. 2, pp. 275-281, 2016. 